Supporting Information for

\title{
Catalysis with a Skip: Dynamically Coupled Addition, Proton \\ Transfer, and Elimination During Au- and Pd-Catalyzed Diol
}

\section{Cyclization}

\author{
Matthew S. Teynor, Windsor Scott, and Daniel H. Ess* \\ Department of Chemistry and Biochemistry, Brigham Young University, Provo, Utah 84602 \\ *dhe@chem.byu.edu
}

Table of Contents

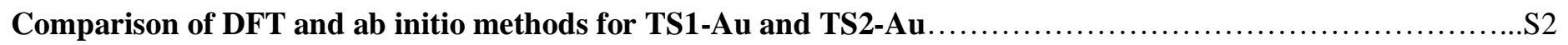

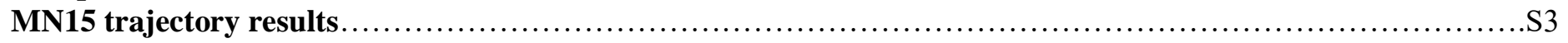

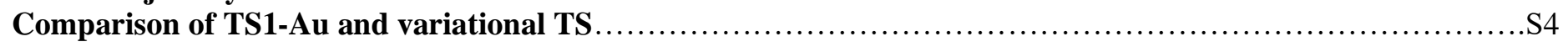

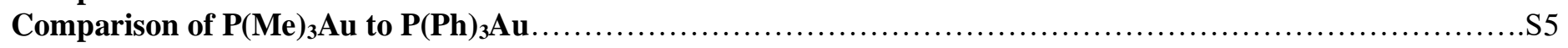


Comparison of DFT and ab initio methods for TS1-Au and TS2-Au
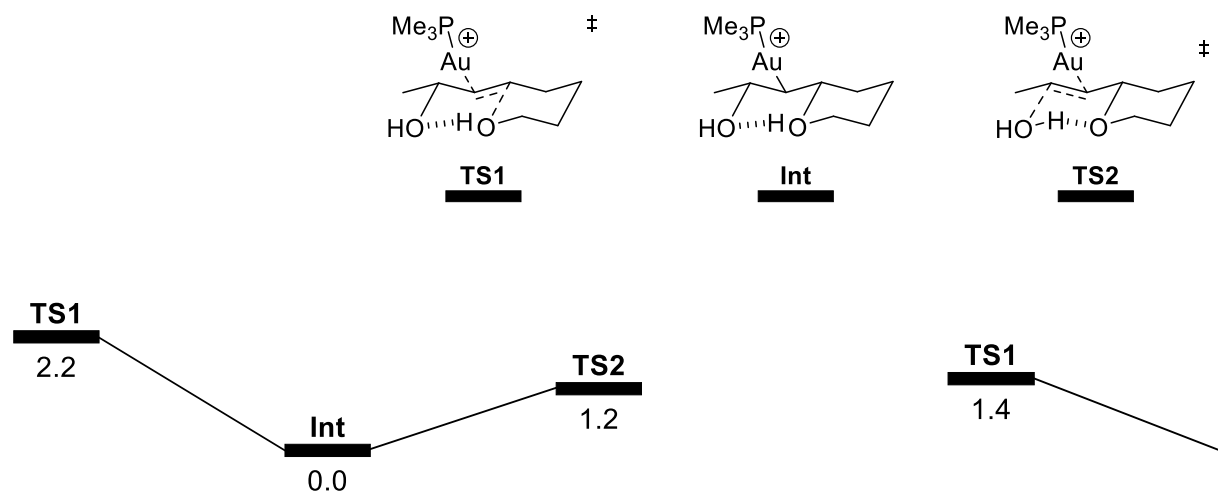

M06/6-31G**[LANL2DZ for Au]

SMD Solvent: Dichloromethane

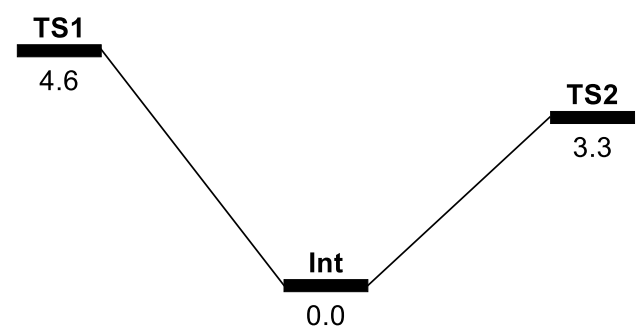

${ }^{a}$ DLPNO-CCSD(T)/def2-TZVP No Solvent

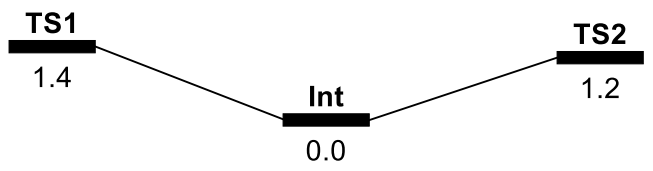

M06/def2-TZVP

SMD Solvent: Dichloromethane

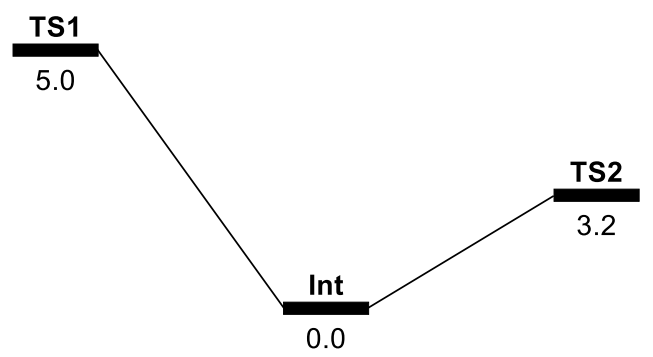

a)DLPNO-CCSD(T)/def2-TZVP

SMD Solvent: Dichloromethane

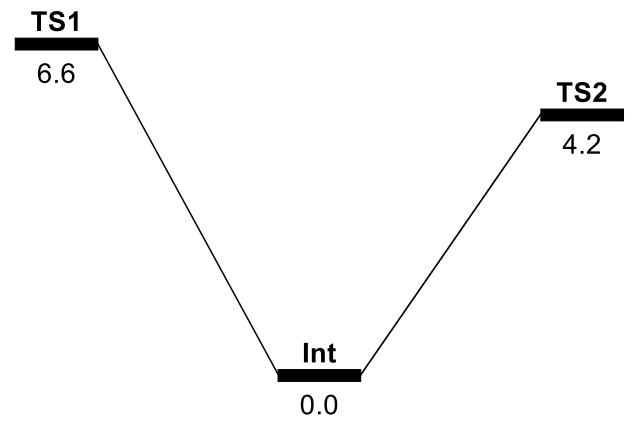

MN15/6-31G**[LANL2DZ for Au]

SMD Solvent: Dichloromethane

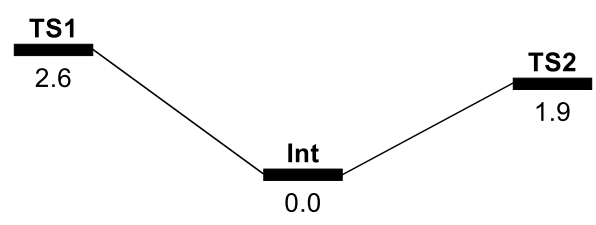

${ }^{\text {a) }} \mathrm{M} 06 / 6-31 \mathrm{G}^{* *}$ [LANL2DZ for $\left.\mathrm{Au}\right]$ No Continuum Solvent

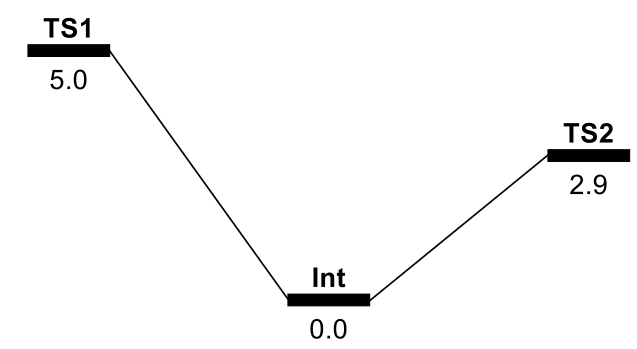

$\omega \mathrm{B} 97 \mathrm{X}-\mathrm{D} / 6-31 \mathrm{G}^{* *}$ [LANL2DZ for $\left.\mathrm{Au}\right]$

SMD Solvent: Dichloromethane

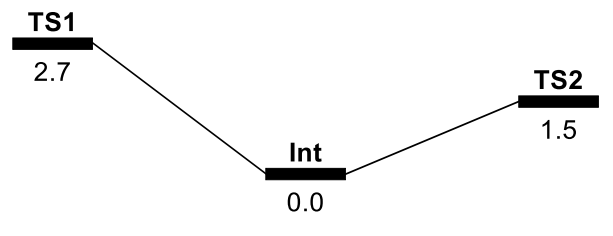

${ }^{\text {a) }}$ M06/6-31G** [LANL2DZ for $\left.\mathrm{Au}\right]$

CPCM Solvent: Dichloromethane

Figure S1. Comparison of energy landscapes with DFT and ab initio methods. Electronic energies are given in kcal/mol. a) Single point calculations using M06/6-31G**[LANL2DZ for Au], SMD Solvent: Dichloromethane structures. 


\section{MN15 trajectory results}

While M06 and DLPNO-CCSD(T) results are very similar, we wondered what would change in Au trajectories in a deeper, more stabilized Au-alkyl intermediate was found. The MN15 functional, compared to DLPNO-CCSD(T), overestimates the stability of the Au-alkyl intermediate. Therefore, we ran a small set of test trajectories starting from TS1-Au to determine the impact of a deeper energy well. Figure S2 shows that the deeper well results in the ballistic trajectories becoming unrelaxed trajectories. Importantly, all non-recrossing trajectories skipped the Au-alkyl intermediate with the reaction steps coupled.

\section{$\underline{\text { Breaking } \mathrm{C}-\mathrm{O} \text { bond }}$}

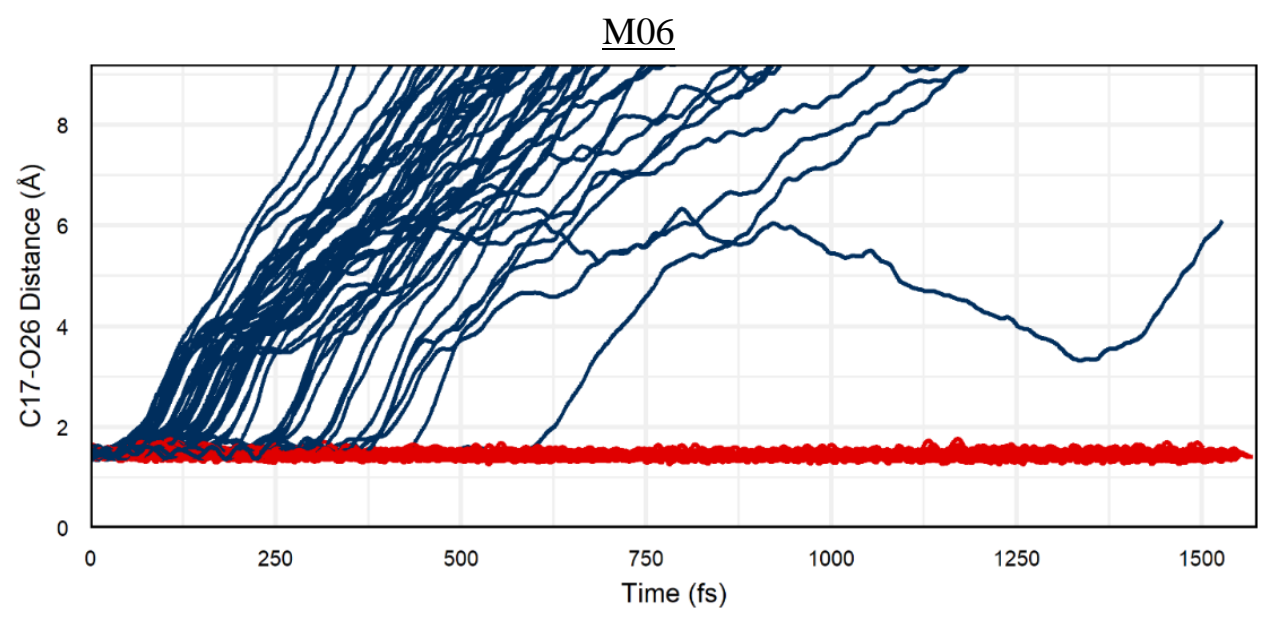

$\underline{\text { MN15 }}$

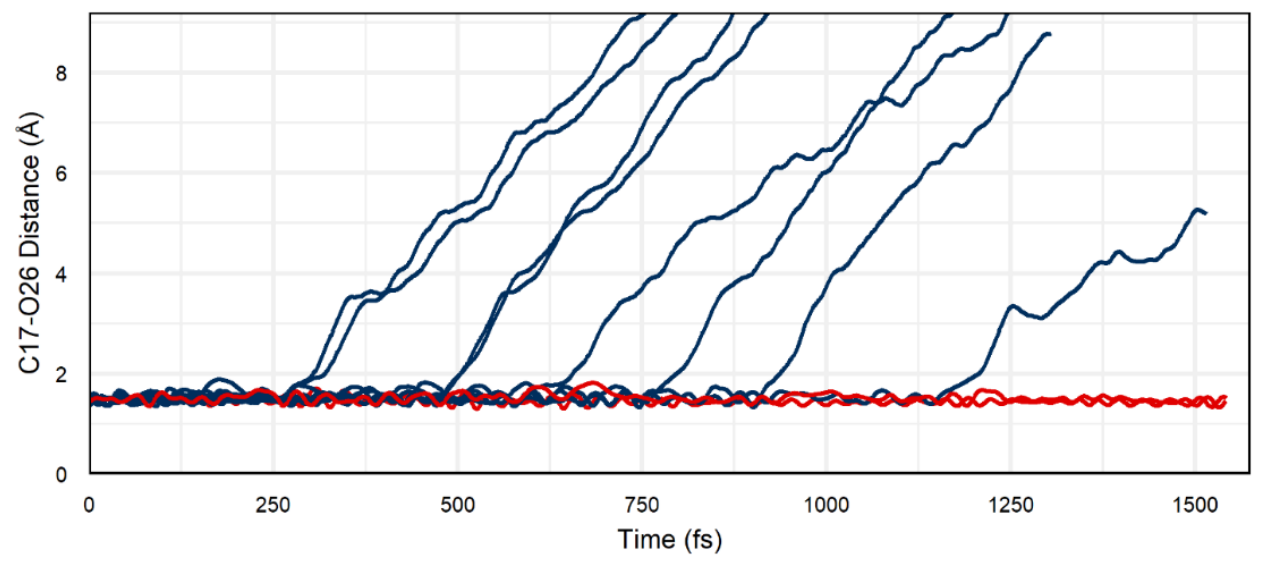

Figure S2. Comparison of M06 and MN15 trajectories progressing forward from TS1-Au. 10 trajectories are displayed for MN15/6-31G**[LANL2DZ for Au]. 


\section{Comparison of Au-TS1 and variational TS}

We calculated the variational transition state structure with the M06 functional and the 6-31G**[LANL2DZ for $\mathrm{Au}$ ] basis set, using Polyrate, Gaussian 16, and the Gaussrate interface. The variational transition state has a nearly identical energy ( $0.08 \mathrm{kcal} / \mathrm{mol}$ lower) and structure to TS1-Au. Figure S3 shows a comparison of key bonds lengths.

a)

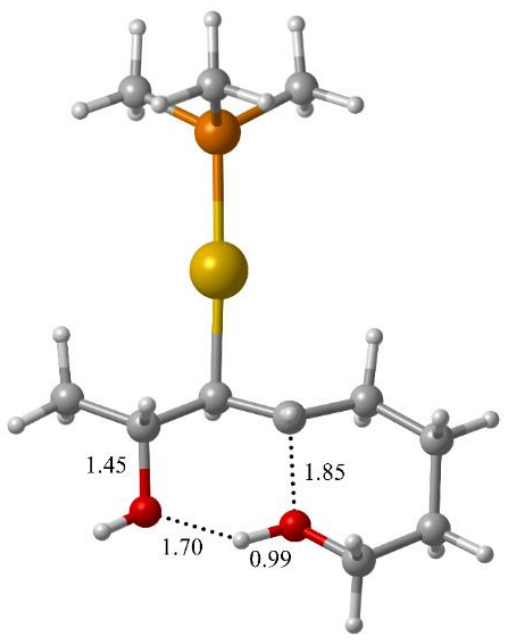

b)

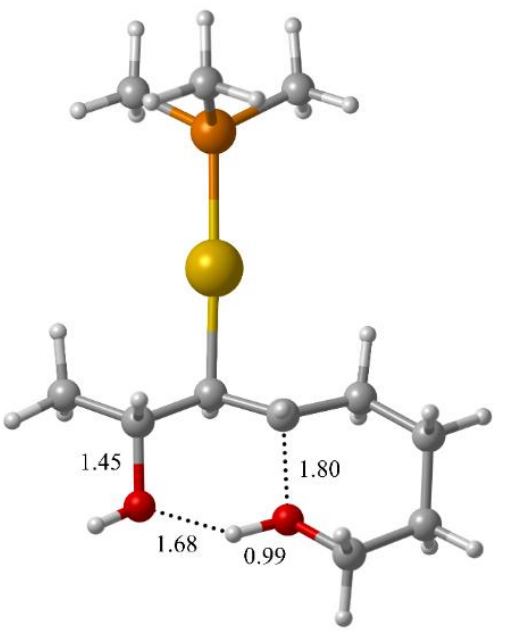

Figure S3. Comparison of TS1-Au (a) and the variational transition state (b). Key bond lengths are labeled in angstroms. 


\section{Comparison of $\mathrm{P}(\mathrm{Me})_{3} \mathrm{Au}$ to $\mathrm{P}(\mathrm{Ph})_{3} \mathrm{Au}$}

We calculated the energy landscape for the Au reaction replacing $\mathrm{PMe}_{3}$ with $\mathrm{PPh}_{3}$ with the $\mathrm{M} 06$ functional and the 6-31G** [LANL2DZ for $\mathrm{Au}$ ] basis set. The $\mathrm{PPh}_{3}$ energy landscape is very similar to the corresponding landscape for $\mathrm{PMe}_{3}$, as shown in Figure S4. We launched 15 trajectories from TS1-Au of the PPh) $)_{3}$ surface. Figure S5 shows that similar to $\mathrm{PMe}_{3}$ all non-recrossing trajectories skipped the Au-alkyl intermediate with the reaction steps coupled.

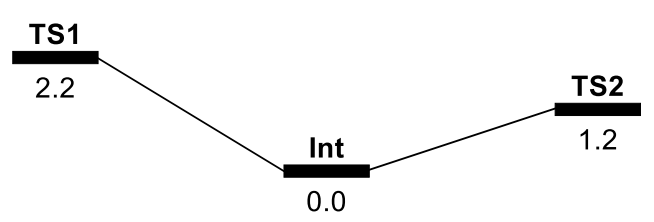

$\mathrm{P}(\mathrm{Me})_{3}$

M06/6-31G**[LANL2DZ for Au]

SMD Solvent: Dichloromethane

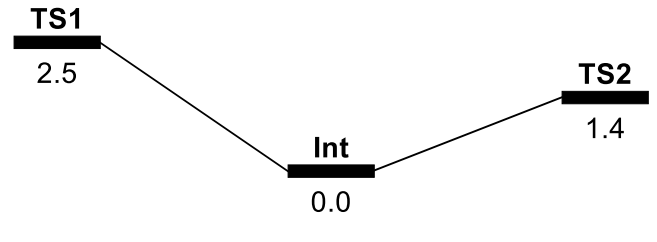

$\mathrm{P}(\mathrm{Ph})_{3}$

M06/6-31G**[LANL2DZ for Au]

SMD Solvent: Dichloromethane

Figure S4. Comparison of energy landscapes with $\mathrm{P}(\mathrm{Me})_{3}$ and $\mathrm{P}(\mathrm{Ph})_{3}$. Electronic energies are given in $\mathrm{kcal} / \mathrm{mol}$.

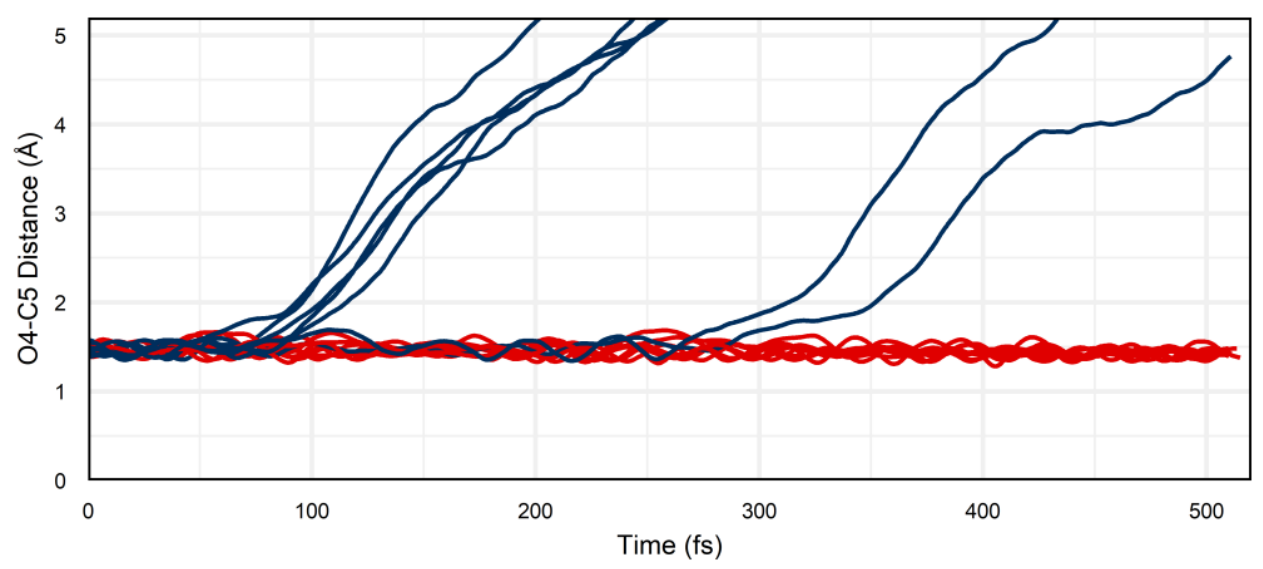

Figure S5. Breaking C-O bond distance for 15 trajectories progressing forwards from TS1-Au on the $\mathrm{P}(\mathrm{Ph})_{3}$ surface using M06/6-31G**[LANL2DZ for Au]. 